\title{
Underlying Event Studies and Forward Physics at CMS
}

\author{
Paolo Bartalini* \\ NTU \\ E-mail: paolo.bartaliniecern.ch \\ on behalf of the CMS collaboration
}

This paper summarizes the early underlying event and forward measurements of the CMS collaboration using pp collision data up to highest centre of mass energies of $7 \mathrm{TeV}$. These studies constitute important tests of the Standard Model and inputs for Monte Carlo tuning.

The underlying event in pp interactions is studied measuring the charged multiplicity density and the charged energy density in the transverse region, which is defined considering the azimuthal distance of the reconstructed tracks with respect to the leading track or leading track-jet of the event, the latter being defined from tracks according to different jet clustering algorithms. In addition, we present the measurement of the underlying event using the jet-area/median approach, demonstrating its sensitivity to different underlying event scenarios.

The energy flow in the forward direction is measured for minimum bias events and events having a dijet system in the central region. In addition, the absence of energy deposition in the forward region is used to observe diffractive events.

We compare our underlying event and forward results with predictions from Monte Carlo event generators tuned to describe the charged particle spectra seen at central rapidities.

35th International Conference of High Energy Physics - ICHEP2010,

July 22-28, 2010

Paris France

\footnotetext{
* Speaker.
} 
This paper summarizes the early "underlying event" (UE) and forward measurements of the CMS collaboration using pp collision data up to highest energies of $\sqrt{s}=7 \mathrm{TeV}$. A detailed description of the detector is available in Ref. [1]. Monte Carlo (MC) predictions are compared, after full detector simulation, to the data without any correction for detector effects. The predictions for inelastic events are calculated using several tunes of the PYTHIA program, versions 6.420 [2, 3] and $8.135[4,5]$. PHOJET [6] is also used in the forward measurements. The following PYTHIA-6 tunes are used: D6T [7, 8], DW [8], Perugia-0 (P0) [9], and CW, the last being adapted from the DW tune as described in Ref. [10]. Default PYTHIA-8 and PHOJET tunes are used.

\section{Early underlying event measurements at CMS}

In the presence of a hard process, characterized by particles or clusters of particles with a large transverse momentum $p_{T}$ with respect to the beam direction, the final state of hadron-hadron interactions can be described as the superposition of several contributions: products of the partonic hard scattering with the highest $p_{T}$, including initial and final state radiation; hadrons produced in additional "multiple parton interactions" (MPI); and "beam-beam remnants" (BBR) resulting from the hadronization of the partonic constituents that did not participate in other scatterings. Products of MPI and BBR form the UE, which cannot be separated from initial and final state radiation.

The early CMS UE measurements focus on the understanding of the UE dynamics studying charged particle production with two different approaches. The first (traditional) approach [10, 11] concentrates on the study of the transverse region, which is defined considering the azimuthal distance of the reconstructed tracks with respect to the leading track or leading track-jet of the event: $60^{\circ}<|\Delta \phi|<120^{\circ}$. The jet reconstruction algorithm used in these studies is SisCone [13]. On top of the traditional approach, a new methodology using anti- $k_{T}$ jets [14] and relying on the measurement of their area [15] is adopted for the first time by CMS using charged particles in pp collision data collected at $\sqrt{s}=0.9 \mathrm{TeV}$ [12]. The new set of UE observables consider the whole pseudorapidity-azimuth plane instead of the transverse region and inherently take into account the leading jets of an event.

The centre-of-mass energy dependence of the hadronic activity in the transverse region is presented in the left panel of Fig. 1 as a function of the $p_{T}$ of the leading track-jet. The data points represent the average multiplicity dependence, for $\sqrt{s}=0.9 \mathrm{TeV}$ and $\sqrt{s}=7 \mathrm{TeV}$ using tracks with a pseudorapidity $|\eta|<2.0$ and $p_{T}>0.5 \mathrm{GeV} / \mathrm{c}$. A significant growth of the average multiplicity and of the average scalar $p_{T}$ sum of charged particles transverse to that of the leading track-jet is observed with increasing scale provided by the leading track-jet $p_{T}$, followed by saturation at large values of the scale. A significant growth of the activity in the transverse region is also observed, for the same value of the leading track-jet $p_{T}$, from $\sqrt{s}=0.9 \mathrm{TeV}$ to $\sqrt{s}=7 \mathrm{TeV}$. These observations are consistent with the ones obtained at Tevatron [16].

The central panel of Fig. 1 presents, for charged particles in the transverse region, the the normalized $\sum p_{T}$ distribution. Events are selected with a minimal value of the leading track-jet $p_{T}>20 \mathrm{GeV} / \mathrm{c}$. The particle $p_{T}$ spectrum (not shown) extends up to $p_{T}>10 \mathrm{GeV} / \mathrm{c}$, indicating the presence of a hard component in particle production in the transverse region. A hardening of the multiplicity distribution, of the scalar $p_{T}$ sum distribution and of the charged particle $p_{T}$ spectrum is also observed with increasing scale values. The distributions for track-jet $p_{T}>20 \mathrm{GeV} / \mathrm{c}$, which 

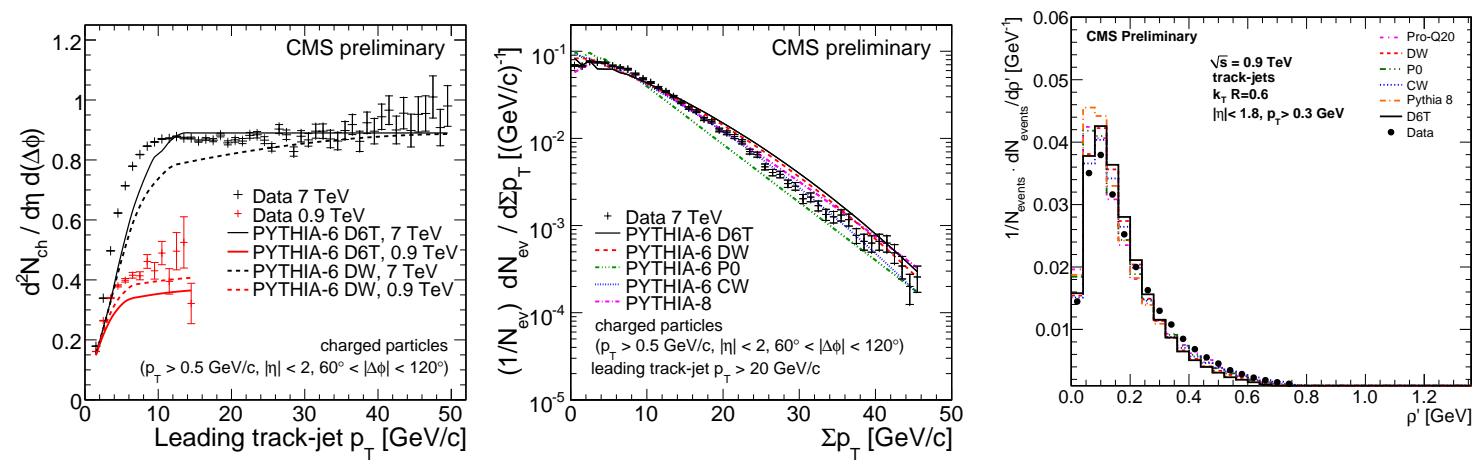

Figure 1: (Left plot) average multiplicity in the transverse region as a function of the leading track-jet $p_{T}$, for data at $\sqrt{s}=0.9 \mathrm{TeV}$ and $\sqrt{s}=7 \mathrm{TeV}$. (Central plot) normalized scalar $\sum p_{T}$ distribution in the transverse region for data at $\sqrt{s}=7 \mathrm{TeV}$; the leading track-jet is required to have $p_{T}>20 \mathrm{GeV} / \mathrm{c}$. (Right plot) normalized median of $p_{T}$ over jet area for track-jets reconstructed from collision data at $\sqrt{s}=0.9 \mathrm{TeV}$ (black circles). Predictions from the investigated MC tunes, with full detector simulation, are compared to the data. The inner error bars indicate the statistical uncertainties affecting the measurements; for convenience systematic uncertainties on MC predictions are presented in the form of systematic uncertainties on the data points; the outer error bars thus represent the statistical uncertainties on the measurements and the systematic uncertainties affecting the MC predictions added in quadrature.

extend up to quite large values of the selected observables in the transverse region $\left(N_{\mathrm{ch}}\right.$ up to 40 , $\sum p_{T}$ up to $50 \mathrm{GeV} / \mathrm{c}$ ), are quite well described by the various MC models, over several orders of magnitude. This observation gives support to the implementation of MPI in PYTHIA.

The novel technique to quote the UE activity [15] relies on the introduction of "ghosts", virtual deposits of very low energy filling the overall phase space which are taken into account by the jet clustering algorithm. The estimator of the overall soft background activity in an event can be derived as the median of the ratio between the transverse momentum and the area of the jets. One of the advantages of using the median compared to the mean is that it turns out to be less sensitive to the influence of outliers, i.e. in particular the leading jets in an event. In order to cope with the low occupancy observed at $\sqrt{s}=0.9 \mathrm{TeV}$, CMS redefines such observable restricting the median only to those jets which have physical deposits on top of ghosts:

$$
\rho^{\prime}=\operatorname{median}\left[\left\{\frac{p_{\mathrm{T}_{j}}}{A_{j}}\right\}\right] \cdot C
$$

where $C$ is the occupancy of the event, which is the summed area $\sum_{j} A_{j}$ covered these jets divided by the considered detector region $A_{\text {tot }}$. In the right panel of Fig. 1 the $\rho^{\prime}$ observable is presented for minimum bias events. Jets are reconstructed from tracks with $|\eta|<2.0$ and $p_{T}>0.3 \mathrm{GeV} / \mathrm{c}$. The general pattern of deviations from data with respect to the considered PYTHIA tunes looks rather similar to the one observed with the previous methodology.

\section{Early forward measurements at CMS}

CMS reports a measurement of the energy flow in the forward region $(3.15<|\eta|<4.9$, where 
$\eta$ denotes the pseudorapidity) [17] for minimum bias and dijet events in pp interactions with centreof-mass energies $\sqrt{s}$ of $0.9 \mathrm{TeV}, 2.36 \mathrm{TeV}$ and $7 \mathrm{TeV}$. This measurement is connected to the ones reported in the previous section as the basic philosophy is the same: it concentrates on the complementary activity of a pp interaction for different energy scales of the reconstructed leading objects.

The energy flow in the region of the Hadron Forward detetor is measured in two different event classes: in minimum bias events and in events with a hard scale provided by a dijet system at central pseudorapidities $(|\eta|<2.5)$ and with transverse energy $E_{T, j e t}>8 \mathrm{GeV}$ for $\sqrt{s}=0.9 \mathrm{TeV}$ and $2.36 \mathrm{TeV}$; the dijet threshold is increased to $20 \mathrm{GeV}$ for $\sqrt{s}=7 \mathrm{TeV}$. The results are qualitatively similar at all the investigated centre of mass energies. Fig. 2 shows the results of the forward energy flow at $\sqrt{s}=7 \mathrm{TeV}$ for the two event classes compared to predictions from Monte Carlo event generators that are tuned to describe the charged particle spectra seen at central rapidities. The measured forward energy flow is found to be significantly different between the two event classes, with a sensitive increase and a more central activity seen in dijet events.
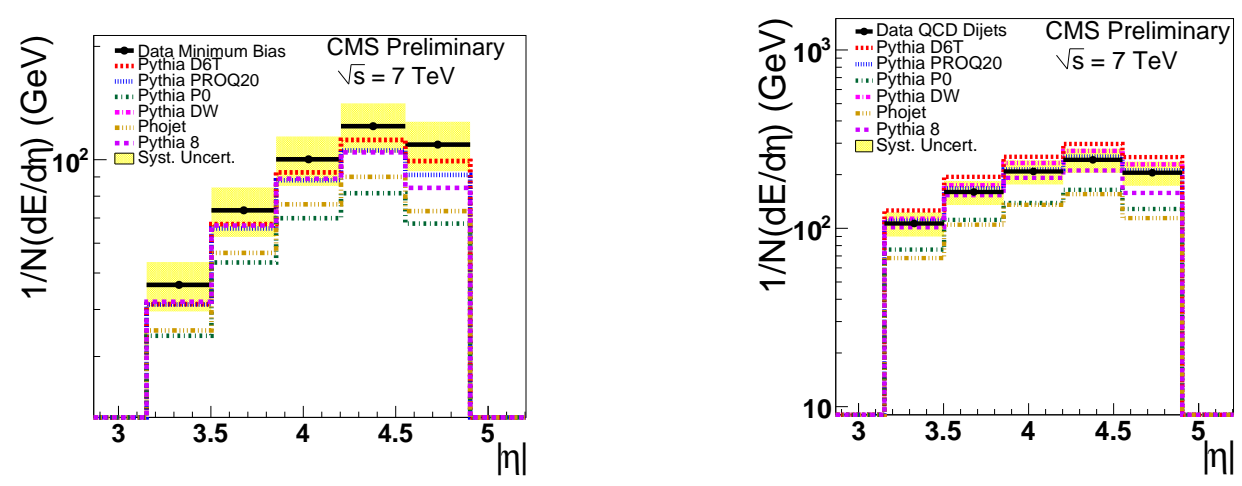

Figure 2: Energy flow in the minimum bias (left) and di-jet (right) samples as a function of $\eta_{\text {obs }}$ at $\sqrt{s}=7$ $\mathrm{TeV}$. Uncorrected data are shown as points, the histograms correspond to the MC predictions. Error bars corresponds to statistical errors. The shaded bands in these plots represent the systematic uncertainties of the measurements, which are largely correlated point-to-point.

CMS presents the observation of a diffractive signal [18] dominated by the inclusive singlediffractive (SD) reaction $p p \rightarrow X p$ as well as a comparison of the data with some of the investigated MC models. Figure 3 shows the distribution of minimum bias data at $\sqrt{s}=0.9 \mathrm{TeV}$ and $\sqrt{s}=2.36$ as a function of $E \pm p_{z}=\sum\left(E_{i} \pm p_{z, i}\right)$, where the sum runs over all calorimeter towers, including the ones in the forward region. The longitudinal momentum $p_{z}$ is calculated as $E \cos \theta$, where $E$ is the tower energy and $\theta$ is the angle between the $z$ axis and the direction defined by the center of the tower and the nominal interaction point. Energy and longitudinal momentum conservation can be used to show that this variable approximately equals twice the Pomeron energy; the plus (minus) sign applies to the case in which the proton emitting the Pomeron moves in the $+z(-z)$ direction. Diffractive events cluster at very small values of $E \pm p_{z}$, reflecting the peaking of the cross section at small $\xi$ (longitudinal momentum loss of diffractively scattered proton). Since it is not possible, on an event-by-event basis, to determine which is the proton emitting the Pomeron, the distributions from the data contain an admixture of "wrong-sign" events for which the proportionality between $\xi$ and $E \pm p_{z}$ does not hold. The bands illustrate the effect of a $10 \%$ energy scale uncertainty in 
the calorimeters and should be taken as a rough estimate of the systematic uncertainty due to the current imperfect understanding and simulation of the detector. A clear diffractive contribution is evident. Cross checks with the help of additional variables sensitive to diffraction were performed and confirm the results.
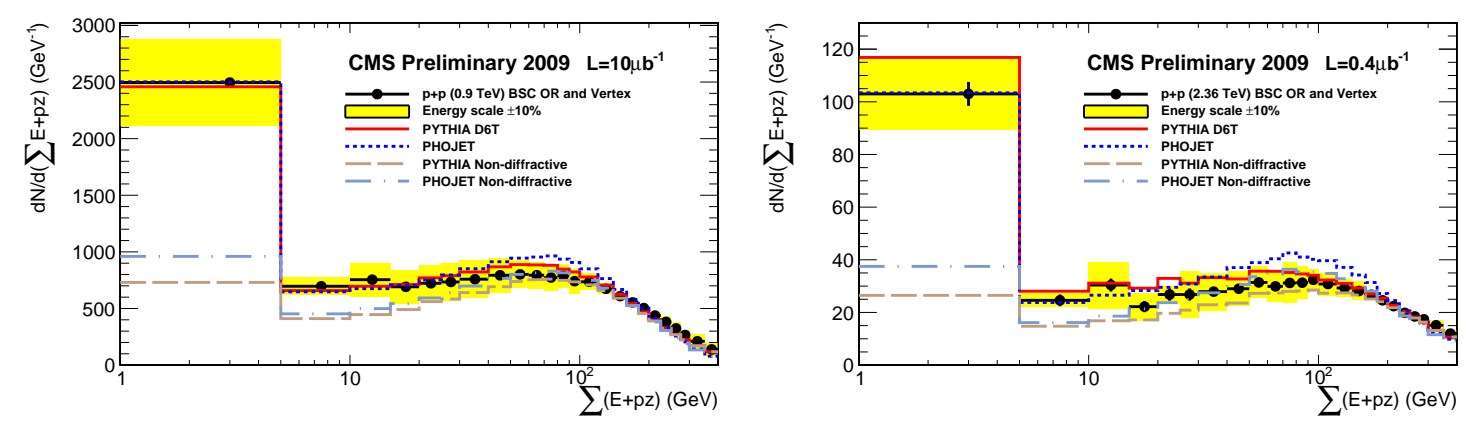

Figure 3: Distributions of minimum bias events as a function of $E+p_{z}$ at $\sqrt{s}=0.9 \mathrm{TeV}$ and $\sqrt{s}=2.36$ $\mathrm{TeV}$. The distributions are uncorrected. The MC predictions are also shown, normalised to the data. The vertical bars indicate the statistical uncertainty of the data. The bands illustrate the effect of a $10 \%$ energy scale uncertainty in the calorimeters.

\section{References}

[1] CMS Collaboration, JINST 3 (2008) S08004.

[2] T. Sjostrand and M. van Zijl, Phys. Lett. B 188, 149 (1987).

[3] T. Sjostrand, S. Mrenna and P. Z. Skands, JHEP 0605, 026 (2006) [arXiv:hep-ph/0603175].

[4] T. Sjostrand, [arXiv:0809.0303 [hep-ph]].

[5] R. Corke, [arXiv:0901.2852 [hep-ph]].

[6] F. W. Bopp, R. Engel and J. Ranft, arXiv:hep-ph/9803437.

[7] R. Field, Acta Phys. Polon. B 39 (2008) 2611.

[8] R.. Field, in "Proceedings of the First MPI@LHC," [arXiv:1003.4220 [hep-ex]].

[9] P. Z. Skands, [arXiv:0905.3418 [hep-ph]].

[10] CMS Collaboration, [arXiv:1006.2083 [hep-ex]].

[11] CMS Collaboration, CMS-PAS-QCD-10-010.

[12] CMS Collaboration, CMS-PAS-QCD-10-005.

[13] G. P. Salam and G. Soyez, JHEP 0705 (2007) 086 [arXiv:0704.0292 [hep-ph]].

[14] M. Cacciari, G. P. Salam and G. Soyez, JHEP 0804, 063 (2008) [arXiv:0802.1189 [hep-ph]].

[15] M. Cacciari, G. P. Salam and S. Sapeta, JHEP 1004 (2010) 065 [arXiv:0912.4926 [hep-ph]].

[16] A. A. Affolder et al. [CDF Collaboration], Phys. Rev. D 65 (2002) 092002.

[17] CMS Collaboration, CMS-PAS-FWD-10-002.

[18] CMS Collaboration, CMS-PAS-FWD-10-001. 